\title{
El aprendizaje expresivo y las diferencias de género en la Educación Superior de Música
}

\section{Expressive learning and gender differences in Music Higher Education}

\author{
Carolina Bonastre \\ cbonastre@ucm.es \\ Departamento de Didáctica de las Lenguas, Artes y Educación Física \\ Universidad Complutense de Madrid \\ Madrid, España \\ ORCID: https://orcid.org/0000-0001-8632-5655
}

\author{
Roberto Nuevo \\ roberto.nuevob@gmail.com \\ Instituto de Salud Carlos III \\ Madrid, España \\ ORCID: https://orcid.org/0000-0003-3385-2747
}

doi: 10.7203/LEEME.44.15679

Recibido: 29-07-2019 Aceptado: 11-10-2019. Contacto y correspondencia: Carolina Bonastre, Dpto. de Dtca. de las Lenguas, Artes y Educ. Física Universidad Complutense de Madrid, C/Rector Royo Villanova 1, 28040. Madrid. España.

\section{Resumen}

En un momento en el que la igualdad entre mujeres y hombres se considera necesaria en la mayoría de las culturas occidentales, el resultado de las investigaciones en el área de música muestra que se siguen produciendo diferencias de género. Lo cual afecta a aspectos como: a) la elección instrumental de los estudiantes; y b) las salidas profesionales, principalmente en composición y dirección orquestal. Desde un enfoque educativo se plantea si se observa un cambio en la actualidad de estos aspectos y si existen también diferencias de género en el uso de estrategias de enseñanza-aprendizaje de la expresividad musical. 196 alumnos ingleses y españoles de enseñanzas superiores aportaron información al respecto. Los resultados indican que persisten las diferencias en estos aspectos generales y que las mujeres respecto a los hombres consideran más relevante el uso de estrategias como las metáforas e imágenes.

Palabras clave: Educación música, mujeres y hombres, estudios de género, Enseñanzas Superiores.

\section{Abstract}

At a time when equality between women and men is considered necessary in most Western cultures, the result of investigations in the area of music shows that gender differences are still present. That affects aspects such as: a) the students' instrumental choice; and b) career opportunities, mainly regarding composition and orchestral conducting. From an educational approach this paper considers whether a change in the current situation of these aspects is observable and if there are gender differences in the utilization of strategies of teaching and learning of musical expressivity. 196 English and Spanish students of Higher Education provided information regarding this situation, and results indicate that differences persist in these general aspects and that women compared to men consider more relevant the utilization of strategies such as metaphors and images.

Key words: Music education, women and men, gender studies, teaching and learning, Higher Education.

@CAROLINA BONASTRE Y ROBERTO NUEVO. THE CONTENT OF THIS ARTICLE IS THE SOLE RESPONSIBILITY OF THE AUTHORS. THE REVISTA ELECTRÓNICA DE LEEME AND UNIVERSITAT DE VALĖNCIA ARE NOT LIABLE FOR ANY LEGAL ACTIONS THAT MAY ARISE INVOLVING THE ARTICLE'S CONTENT. REVISTA ELECTRÓNICA DE LEEME -LISTA ELECTRÓNICA EUROPEA DE MÚSICA EN LA EDUCACIÓN-HTTP://OJS.UV.ES/INDEX/PHP/LEEME/INDEX. ISSN: 1575-9563. EDITORES: UNIVERSIDAD DE VALENCIA Y JESÚS TEJADA GIMÉNEZ. VISIBILIDAD DE ESTA REVISTA: SCOPUS, EMERGING SOURCES CITATION INDEX (CLARIVATE), EBSCO, CINDOC (CSIC), CITEFACTOR, COPAC, DIALNET, DICE (CSIC), DOAJ, E-REVISTAS ESTA PUBLICADA CON EL APOYO INSTITUCIONAL DE REDIRIS-CONSEJO SUPERIOR DE INVESTIGACIONES CIENTIFICAS Y ES DE ACCESO LIBRE. CREATIVE COMMONS LICENSE 4.O BY
B. 


\section{Introducción}

La música es una fuente de expresión artística y un vehículo de comunicación emocional. La expresividad musical es considerada relevante para distintos elementos del proceso comunicativo como el público o los propios intérpretes, que muestran su interés por alcanzar este objetivo, del mismo modo que los compositores (Juslin y Laukka, 2004; Lindström, Juslin, Bressin y Williamon, 2003). Por tanto, es importante indagar en el aprendizaje de este aspecto en el arte sonoro.

\subsection{El aprendizaje de la expresividad musical}

En estos últimos años se han propuesto diferentes proyectos de mejora de habilidades expresivas musicales (Sloboda, Gayford y Minassian, 2003; Juslin, Karlsson, Lindström, Friberg y Schoonderwaldt, 2006) mediante el entrenamiento específico o la retroalimentación cognitiva. En esta línea, algunas investigaciones se han centrado en cuáles son las estrategias utilizadas para su aprendizaje. La estrategia más habitual es la instruccional, sumida en el discurso del profesor (Young, Burwell y Pickup, 2003), y de ello dependerá la enseñanza explícita de la expresividad, dado que existen opiniones del profesorado de que las instrucciones verbales sobre factores específicos pueden alcanzar un resultado no natural o una expresividad simulada (Williamon, 2014). No hay que olvidar que la elección de una estrategia u otra del docente está influida por sus opiniones respecto a la propia naturaleza de la expresividad musical (Brenner y Strand, 2013) y esto se refleja directamente en la práctica (Juslin y Persson, 2002).

En torno al uso de estrategias determinadas sobre el aprendizaje de la expresividad, Woody (2006) coteja diferentes opciones: modelado, pautas verbales y uso de metáforas e imágenes. Este autor concluye que la eficacia de éstas es similar, con algunos matices: la primera favorece la consistencia en los resultados, pero supone mejoras leves; la siguiente obtiene cambios más persistentes, pero no siempre en la dirección recomendada; y en los resultados de la última la eficacia varía según el nivel de ejecución del estudiante. Otra aproximación similar fue la de Juslin et al. (2006) que plantea cuatro estrategias básicas utilizadas por el docente: a) modelado que parte de la imitación; b) metáforas o imágenes con el uso de estas herramientas; c) emociones propias dirigiendo al estudiante hacia sus sensaciones emotivas al tocar; y d) técnica que aporta instrucciones específicas para producir cambios sonoros en la ejecución expresiva. En la práctica diaria se considera que se emplean diferentes modelos, combinados o no (Meissner y Timmers, 2019), en función de los objetivos planteados y las necesidades del estudiante.

@CAROLINA BONASTRE Y ROBERTO NUEVO. THE CONTENT OF THIS ARTICLE IS THE SOLE RESPONSIBILITY OF THE AUTHORS. THE REVISTA ELECTRÓNICA DE LEEME AND UNIVERSITAT DE VALĖNCIA ARE NOT LIABLE FOR ANY LEGAL ACTIONS THAT MAY ARISE INVOLVING THE ARTICLE'S CONTENT. REVISTA ELECTRÓNICA DE LEEME -LISTA ELECTRÓNICA EUROPEA DE MÚSICA EN LA EDUCACIÓN-HTTP://OJS.UV.ES/INDEX/PHP/LEEME/INDEX. ISSN: 1575-9563. EDITORES: UNIVERSIDAD DE VALENCIA Y JESÚS TEJADA GIMÉNEZ. VISIBILIDAD DE ESTA REVISTA: SCOPUS, EMERGING SOURCES CITATION INDEX (CLARIVATE), EBSCO, CINDOC (CSIC), CITEFACTOR, COPAC, DIALNET, DICE (CSIC), DOAJ, E-REVISTAS
(CSIC), EBSCO PREMIER, ERIH+, GALE CENGAGE L EARNING, IN-RECS, IRESIE, LATINDEX, MIAR, OCLC WORLDCAT, RESH, REDIB, RILM CORE JOURNALS, SUDOC, ULRICHS, ESTA REVISTA ESTA PUBLICADA CON EL APOYO INSTITUCIONAL DE REDIRIS-CONSEJO SUPERIOR DE INVESTIGACIONES CIENTIFICAS Y ES DE ACCESO LIBRE. CREATIVE COMMONS LICENSE 4.0 BY 


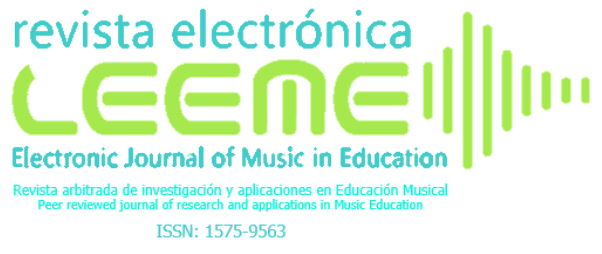

SECCIÓN MONOGRAFICO: CAROLINA BONASTRE Y ROBERTO NUEVO SECCION MONOGRAFICO: CAROLINA BONASTRE Y ROBERTO NUEVO
EL APRENDIZAJE EXPRESIVO Y LAS DIFERENCIAS DE GÉNERO EN LA EDUCACIÓN SUPERIOR DE
MÚSICA (REVISTA ELECTRÓNICA DE LEEME) NÚMERO 44, PP. 87-101
HTTPS://OJS.UV.ES/INDEX.PHP/LEEME/INDEX

Estos aspectos han sido poco tratados desde una perspectiva de género, aunque hay indicios de que podrían existir distinciones significativas entre las preferencias sobre algunas estrategias (Marugán et al., 2011). De hecho, en general, las diferencias de género no suelen ser un predictor relevante en la evaluación de habilidades y conductas musicales (Müllensiefen, Gingras, Musil, y Stewart, 2014), pero sí afectan a las opiniones y elecciones de los estudiantes de música.

\subsection{Diferencias de género en el campo de la música}

A lo largo de la Historia, ha habido diferencias notables en la educación de los hombres y de las mujeres. Ya entrados en el siglo XXI aún se da importancia a estos aspectos porque no se han terminado de limar dichas diferencias (aunque el sesgo es mucho menor). Por ello, es relevante dirigir la mirada a estos aspectos dado que todavía se refleja poca presencia de las mujeres en puestos de responsabilidad o en algunas salidas profesionales. Algo seguramente motivado por causas históricas arraigadas en la cultura, aspectos sociales, o elementos relacionados con identidad y diversidad. Así, los estereotipos existentes se reflejan en la educación en general y en el campo de la música en particular (Soler Campo, 2016). De hecho, se contempla en muchas leyes educativas el deseo de alcanzar la igualdad entre hombres y mujeres. Por ejemplo, en la ley educativa española vigente, Ley Orgánica 8/2013, de 9 de diciembre, para la Mejora de la Calidad Educativa (en adelante LOMCE), se plantea el reto de la sociedad democrática de crear condiciones para que alumnas y alumnos expresen sus talentos y alcancen una educación de calidad cuyo soporte es la igualdad y la justicia social. Del mismo modo, se busca construir un sistema educativo que garantice la igualdad de oportunidades y que haga posible el desarrollo de sus potencialidades, y que puedan "acceder a una formación que permita todo tipo de opciones profesionales y el ejercicio de las mismas". (LOMCE, p. 97887).

Aun así, se encuentran diferencias significativas en algunas áreas como la música en puestos de liderazgo (Soler Campo, 2016) y claras desigualdades de género. En este sentido y con el fin de proporcionar igualdad de oportunidades, en la educación superior se han realizado investigaciones en torno a la formación del profesorado dirigidas a la importancia de la reflexión de aspectos relacionados con el género (Díaz Mohedo, 2005). De hecho, en las últimas décadas comienza a aumentar el número de investigaciones en relación con este aspecto que exploran desde distintos enfoques el área de música. Aunque aún son escasos destacan los estudios en torno al papel de la mujer en la música mediante figuras femeninas concretas o en referencia a la situación de estos últimos años al respecto (De las Heras Fernández y García Gil, 2018; Soler Campo, 2016).

En el Reino Unido, reflejando el papel cambiante de la mujer en la sociedad, se ha estudiado el éxito en los exámenes en diferentes temas y en la mayoría de los niveles, siendo más elevados los resultados femeninos (Department for Education and Skills, 2007). De algún 


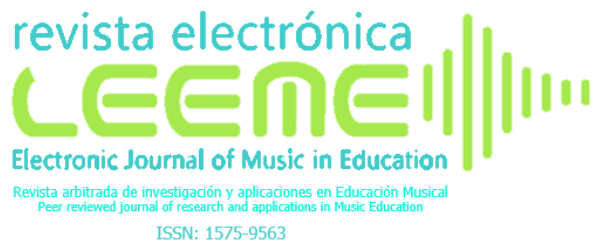

SECCIÓN MONOGRAFICO: CAROLINA BONASTRE Y ROBERTO NUEVO SECCION MONOGRÁFICO: CAROLINA BONASTRE Y ROBERTO NUEVO
EL APRENDIZAJE EXPRESIVO Y LAS DIFERENCIAS DE GÉNERO EN LA EDUCACIÓN SUPERIOR DE
MÚSICA (REVISTA ELECTRÓNICA DE LEEME) NÚMERO 44, PP. 87-101 $\frac{\text { HTTPS://OJS.UV.ES/INDEX.PHP/LEEME/INDEX }}{\text { DOI: } 10.7203 / L E E M E .44 .15679}$

modo esto también sucede en el área de música (Agak, 2002), aunque no hay diferencias de género consistentes en la capacidad musical (Gordon, 1986). En las enseñanzas musicales no se han encontrado diferencias estadísticamente significativas respecto a la motivación para la práctica y el tiempo semanal dedicado (Hallam et al., 2017), pero sí se encontraron diferencias respecto a las opiniones y valoración de la música instrumental, más positiva desde la perspectiva femenina (Eccles, Wigfield, Harold y Blumenfeld, 1993).

En esta línea y en relación con la interpretación de instrumentos se han encontrado diferencias como que las estudiantes tienden a tener más éxito en el estudio instrumental y a escuchar un repertorio más amplio con una gama mayor de estilos musicales que los estudiantes (Green, 1997). En las investigaciones referidas a las preferencias instrumentales existen estereotipos marcados que se acentúan más en los estudiantes adolescentes que en edades tempranas (Zervoudakes y Tanur, 1994). Hay que tener presentes estos resultados dado que en ambos periodos la mayoría realizan la elección instrumental. Por otro lado, en un estudio sobre las percepciones de los estudiantes en relación con aspectos musicales se encontró que destaca positivamente el papel de la improvisación y la composición en los adolescentes masculinos (Green, 1997).

En lo referente a las salidas profesionales, la incorporación de las mujeres a la composición o a la dirección orquestal sigue un proceso lento, se han alcanzado muchos logros, pero aún queda un largo camino por recorrer (Soler Campo, 2016). La información disponible de las organizaciones que representan a compositores indica que aproximadamente sólo el 20\% de los compositores de todo el mundo son mujeres, un porcentaje que además es poco representado en los trabajos de investigación (Parsons y Ravenscroft, 2016). Hay que tener en cuenta que a esto se suma el hecho de que la interpretación musical de compositoras es significativamente menor, algo observable en planes de estudios de educación superior (Bennett, Macarthur, Hope, Goh, y Hennekam, 2018). Además, en estas enseñanzas se desarrolla la composición como especialidad por lo que tienen un papel crítico que desempeñar en esta desigualdad (Macarthur, 2010).

Por todo ello, el principal objetivo de este trabajo era conocer las diferencias entre la valoración de las mujeres y hombres estudiantes de música en educación superior sobre los métodos más adecuados para la enseñanza-aprendizaje de la expresividad. Adicionalmente, es interesante destacar la posible persistencia de un sesgo de género tanto en la elección de la especialidad instrumental como en las perspectivas profesionales de las estudiantes de este nivel educativo (cercanas a afrontar el mundo laboral). El estudio, además, adopta una perspectiva internacional al incluir estudiantes españoles e ingleses; dos países europeos con sistemas educativos y contextos culturales similares, que hacen más generalizables los resultados del estudio. Se considera como hipótesis de partida que el país de la muestra no afectará a los

@CAROLINA BONASTRE Y ROBERTO NUEVO. THE CONTENT OF THIS ARTICLE IS THE SOLE RESPONSIBILITY OF THE AUTHORS. THE REVISTA ELECTRÓNICA DE LEEME AND UNIVERSITAT DE VALĖNCIA ARE NOT LIABLE FOR ANY LEGAL ACTIONS THAT MAY ARISE INVOLVING THE ARTICLE'S CONTENT. REVISTA ELECTRÓNICA DE LEEME -LISTA ELECTRÓNICA EUROPEA DE MÚSICA EN LA EDUCACIÓN-HTTP://OJS.UV.ES/INDEX/PHP/LEEME/INDEX, ISSN: 1575-9563. EDITORES: UNIVERSIDAD DE VALENCIA Y JESÚS TEJADA GIMÉNEZ. VISIBILIDAD DE ESTA REVISTA: SCOPUS, EMERGING SOURCES CITATION INDEX (CLARIVATE), EBSCO, CINDOC (CSIC), CITEFACTOR, COPAC, DIALNET, DICE (CSIC), DOAJ, E-REVISTAS (CSIC), EBSCO PREMIER, ERIH+, GALE CENGAGE LEARNING, IN-RECS, IRESIE, LATINDEX, MIAR, OCLC WORLDCAT, RESH, REDIB, RILM CORE JOURNALS, SUDOC, ULRICHS, ESTA REVISTA ESTÁ PUBLICADA CON EL APOYO INSTITUCIONAL DE REDIRIS-CONSEJO SUPERIOR DE INVESTIGACIONES CIENTIFICAS Y ES DE ACCESO LIBRE. CREATIVE COMMONS LICENSE 4.O BY 


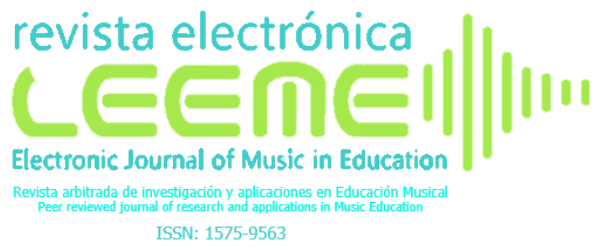
SECCIÓN MONOGRAFICO: CAROLINA BONASTRE Y ROBERTO NUEVO
EL APRENDIZAJE EXPRESIVO Y LAS DIFERENCIAS DE GÉNERO EN LA EDUCACIÓN SUPERIOR DE
MÚSICA (REVISTA ELECTRÓNICA DE LEEME) NÚMERO 44, PP. 87-101
HTTPS://OJS.UV.ES/INDEX.PHP/LEEME/INDEX
DOI: $10.7203 /$ LEEME.44.15679

resultados del estudio y que las características específicas de las mujeres estudiantes coincidirán globalmente entre ambos países, aunque serán diferentes a la de los hombres.

\section{Método}

\subsection{Muestra}

Las características de la muestra de 196 estudiantes, con una media total de edad de 20.70 (Desviación Típica (DT, en adelante)=3.66) son, para cada grupo: a) 117 españoles con una edad comprendida entre 18-35 años y media de 21.03 (DT=3.31), compuesta por un $50.4 \%$ de mujeres (media de edad $=20.84$, DT $=2.95$, rango $=18-30$ ) y un porcentaje un poco menor en hombres (media de edad=21.22, DT=3.65, rango=18-35); b) 79 ingleses entre 18 - 49 años con una edad media de 20.22, (DT=4.08), un $49.4 \%$ de mujeres (media=20.56, DT=5.28) y un $50.6 \%$ de hombres (media $=20.73$, DT=4.03). No hubo diferencias estadísticamente significativas entre ambas muestras ni en edad ni en la proporción de mujeres. El 58\% de los estudiantes estaban en el primer curso, el $31.4 \%$ en $2^{\circ}$, un $6.7 \%$ en $3^{\circ}$, un $1.0 \%$ en $4^{\circ}$ y un $2.1 \%$ eran alumnos de postgrado. No hubo diferencias entre países en función del sexo en la distribución por cursos.

\subsection{Instrumento}

Se elaboró un cuestionario que incluía preguntas generales sobre género, edad, especialidad instrumental y preferencias futuras de líneas de trabajo mediante preguntas dicotómicas o de varias opciones. Se plantearon varias salidas laborales potenciales en el ámbito de la música: docencia, interpretación, composición, dirección, investigación o crítica musical. A continuación, se incluyeron situaciones concretas para ejemplificar las cuatro estrategias planteadas por Juslin et al., (2006). Para ello, se utilizaron preguntas de 4 opciones con una escala tipo Likert sobre el grado de acuerdo o desacuerdo, (rango $=5-20)$, relacionadas con el aprendizaje de cada estrategia. Las cuestiones planteadas se centran en: a) vivencias personales en su aprendizaje; b) uso para la enseñanza; c) corrección global de la estrategia; d) adecuación de la misma para alcanzar un aprendizaje real; e) valoración de esta respecto a otras posibles. Este cuestionario fue avalado en un trabajo anterior (Bonastre y Timmers, 2019).

\subsection{Procedimiento}

Los participantes en esta investigación eran estudiantes de los siguientes centros de educación superior: Faculty of Arts and Humanities (University of Sheffield), Faculty of Performance, Visual Arts and Communications (University of Leeds) y el Conservatorio Superior de Música de Madrid (Madrid). Se elaboró un consentimiento informado que fue

@CAROLINA BONASTRE Y ROBERTO NUEVO. THE CONTENT OF THIS ARTICLE IS THE SOLE RESPONSIBILITY OF THE AUTHORS. THE REVISTA ELECTRÓNICA DE LEEME AND UNIVERSITAT DE VAL ĖNCIA ARE NOT LIABLE FOR ANY LEGAL ACTIONS THAT MAY ARISE INVOLVING THE ARTICLE'S CONTENT. REVISTA ELECTRÓNICA DE LEEME -LISTA ELECTRÓNICA EUROPEA DE MÚSICA EN LA EDUCACIÓN-HTTP://OJS.UV.ES/INDEX/PHP/LEEME/INDEX. ISSN: 1575-9563. EDITORES: UNIVERSIDAD DE VALENCIA Y JESÚS TEJADA GIMÉNEZ. VISIBILIDAD DE ESTA REVISTA: SCOPUS, EMERGING SOURCES CITATION INDEX (CLARIVATE), EBSCO, CINDOC (CSIC), CITEFACTOR, COPAC, DIALNET, DICE (CSIC), DOAJ, E-REVISTAS (CSIC), EBSCO PREMIER, ERIH+, GALE CENGAGE LEARNING, IN-RECS, IRESIE, LATINDEX, MIAR, OCLC WORLDCAT, RESH, REDIB, RILM CORE JOURNALS, SUDOC, ULRICHS, ESTA REVISTA ESTÁ PUBLICADA CON EL APOYO INSTITUCIONAL DE REDIRIS-CONSEJO SUPERIOR DE INVESTIGACIONES CIENTIFICAS Y ES DE ACCESO LIBRE. CREATIVE COMMONS LICENSE 4.O BY 


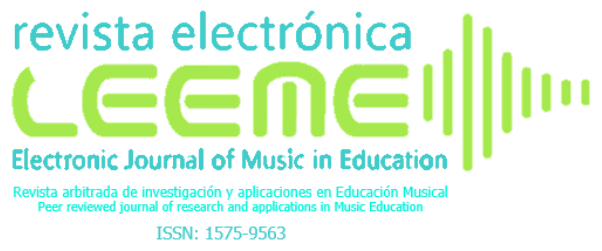
SECCIÓN MONOGRAFICO: CAROLINA BONASTRE Y ROBERTO NUEVO
EL APRENDIZAJE EXPRESIVO Y LAS DIFERENCIAS DE GÉNERO EN LA EDUCACIÓN SUPERIOR DE
MÚSICA (REVISTA ELECTRÓNICA DE LEEME) NÚMERO 44, PP. 87-101
HTTPS:// /OJS.UV.ES/INDEX.PHP/LLEME/INDEX
DOI: $10.7203 /$ LEEME.44.15679

Electronic Journal of Music in Education ISSN: $1575-9563$

aplicado por escrito o verbalmente y la investigación fue aprobada por el comité ético. Los participantes fueron seleccionados mediante muestreo incidental. Se solicitó la participación a los profesores del centro, que a su vez solicitaron la colaboración a los alumnos distribuyendo los cuestionarios. Los profesores recibieron instrucciones precisas de tal modo que remarcaron los objetivos de la investigación, también se recordó que la participación era voluntaria y que se respetaría el anonimato de sus respuestas.

\subsection{Análisis estadístico}

En primer lugar, las proporciones entre ambos géneros se compararon mediante tests chi-cuadrado en diferentes aspectos. Primero, se compararon las pretensiones profesionales de cada uno de los alumnos. A continuación, se comparó entre hombres y mujeres la proporción de estudiantes que se formaban en diferentes especialidades instrumentales. Del mismo modo, se comparó la proporción para cada instrumento específico y también para categorías agrupadas: cuerda frotada, viento-metal, viento-madera, percusión, guitarra, tecla, guitarra eléctrica y voz.

Respecto a las estrategias propuestas sobre expresividad musical, en primer lugar, se realizaron contrastes de normalidad para las puntuaciones totales mediante la prueba de Shapiro-Francia. Las comparaciones entre hombres y mujeres se realizaron mediante pruebas $\mathrm{T}$ de comparación de medias para muestras independientes, en el caso de que se pudiera asumir la normalidad de las distribuciones, y mediante una prueba no paramétrica (U de Mann-Whitney) en caso contrario. Las comparaciones entre ítems específicos se realizaron en todos los casos mediante la prueba U de Mann-Whitney dado el carácter categórico de los datos. Aunque en principio se asumió el criterio convencional de considerar como diferencias significativas entre grupos aquellos contrastes con un valor de $p<.05$, entendiendo que este criterio es necesariamente arbitrario, se resaltan también como relevantes valores cercanos como $p<.07$. La convención de usar valores de $p<.05$ ha sido criticada con frecuencia (p.ej., Cohen, 1994) y un valor conservador de $p<.07$ se sigue usando en estudios recientes como indicador de una tendencia a la significación (por ejemplo, Raglio et al., 2019).

Finalmente, con el fin de controlar el posible efecto de las diferencias entre países en la relación entre el género y los modelos, se realizaron cuatro análisis de regresión lineal. En cada uno de ellos la Variable Dependiente (VD) era la puntuación total en cada modelo de enseñanza-aprendizaje de la expresividad, la Variable Independiente (VI) de interés el género y el país se consideró como covariable. Se incluyó también una constante en cada modelo de regresión de acuerdo a los métodos usuales en este tipo de análisis.

Los análisis indicados se realizaron teniendo en cuenta posibles diferencias en función del curso en el que estaban los estudiantes. Y, todos se realizaron mediante el programa STATA para Windows, versión 13.1.

@CAROLINA BONASTRE Y ROBERTO NUEVO. THE CONTENT OF THIS ARTICLE IS THE SOLE RESPONSIBILITY OF THE AUTHORS. THE REVISTA ELECTRÓNICA DE LEEME AND UNIVERSITAT DE VALĖNCIA ARE NOT LIABLE FOR ANY LEGAL ACTIONS THAT MAY ARISE INVOLVING THE ARTICLE'S CONTENT. REVISTA ELECTRÓNICA DE LEEME -LISTA ELECTRÓNICA EUROPEA DE MÚSICA EN LA EDUCACIÓN-HTTP://OJS.UV.ES/INDEX/PHP/LEEME/INDEX. ISSN: 1575-9563. EDITORES: UNIVERSIDAD DE VALENCIA Y JESÚS TEJADA GIMÉNEZ. VISIBILIDAD DE ESTA REVISTA: SCOPUS, EMERGING SOURCES CITATION INDEX (CLARIVATE), EBSCO, CINDOC (CSIC), CITEFACTOR, COPAC, DIALNET, DICE (CSIC), DOAJ, E-REVISTAS (CSIC), EBSCO PREMIER, ERIH+, GALE CENGAGE LEARNING, IN-RECS, IRESIE, LATINDEX, MIAR, OCLC WORLDCAT, RESH, REDIB, RILM CORE JOURNALS, SUDOC, ULRICHS, ESTA REVISTA ESTÁ PUBLICADA CON EL APOYO INSTITUCIONAL DE REDIRIS-CONSEJO SUPERIOR DE INVESTIGACIONES CIENTIFICAS Y ES DE ACCESO LIBRE. CREATIVE COMMONS LICENSE 4.O BY 


\section{Resultados}

En primer lugar, se encontraron diferencias estadísticamente significativas en función del sexo relativas a la orientación profesional de los estudiantes. Concretamente, los hombres (27.6\%) optaban más que las mujeres $(12.2 \%)$ por dedicarse a la composición $\left(\operatorname{chi}^{2}(1)=7.2\right.$; $p=.007)$ y a la dirección $\left(20.4 \%\right.$ vs. $\left.9.2 \%, \operatorname{chi}^{2}(1)=4.9 ; p=.027\right)$. Respecto a otras áreas como la interpretación, la docencia, la investigación o la crítica musical no se encontraron diferencias relevantes.

Asimismo, hubo un cierto sesgo en función del género en el instrumento principal de los estudiantes: había un mayor porcentaje de mujeres en la especialidad de voz ( $p=.017)$, así como en todas las especialidades de viento-madera $(p=.027)$ excepto en el caso del fagot en el que sucedía lo contrario, aunque el número total de estudiantes de fagot fue pequeño $(n=5)$. Asimismo, hubo un menor porcentaje de mujeres en todas las especialidades de viento-metal ( $p=.007)$, en percusión $(p=.009)$ y en guitarra eléctrica $(p=.043)$. En otras especialidades como piano, guitarra o cuerda, sin embargo, no aparecieron diferencias estadísticamente significativas entre mujeres y hombres. Comparando entre países, el patrón de diferencias fue el mismo, aunque dado el menor tamaño muestral las diferencias fueron menores. Los datos de las especialidades instrumentales en las que se encontraron diferencias estadísticamente significativas se representan gráficamente en la Figura 1.

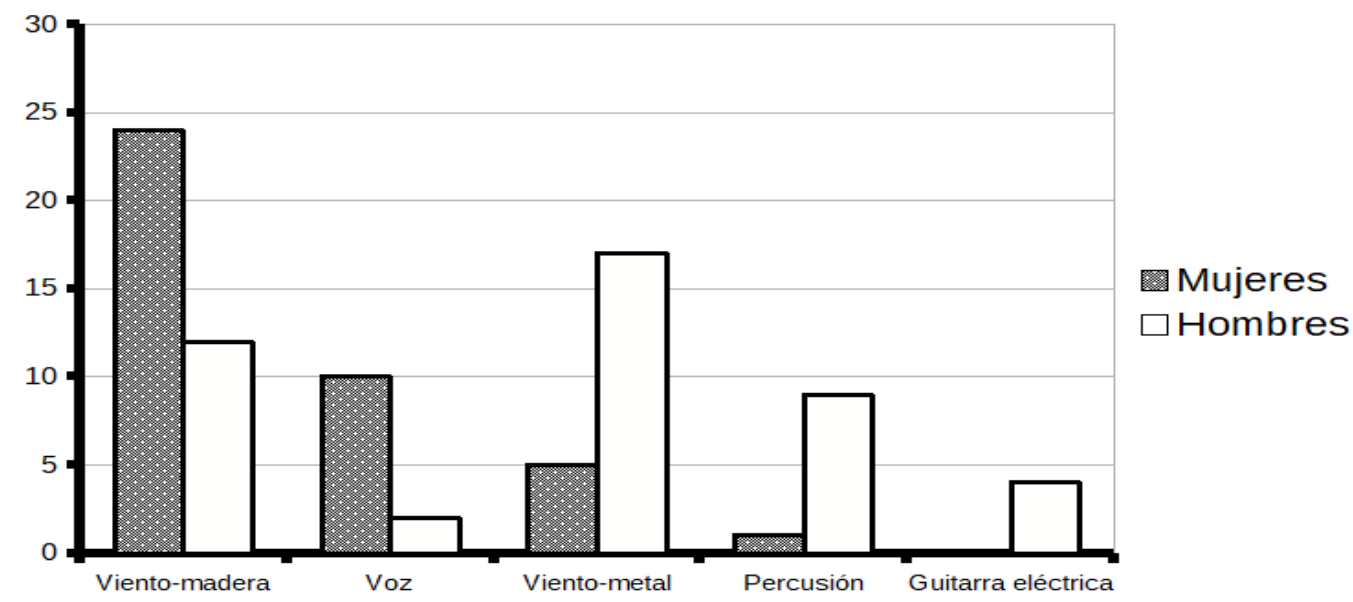

Figura 1. Diferencias entre mujeres y hombres en la especialidad instrumental

En las comparaciones para cada uno de los ítems sobre estrategias para mejorar la expresividad, y las escalas totales entre hombres y mujeres, se encontraron diferencias estadísticamente significativas en tres ítems y la puntuación total de la estrategia de metáforas,

@CAROLINA BONASTRE Y ROBERTO NUEVO. THE CONTENT OF THIS ARTICLE IS THE SOLE RESPONSIBILITY OF THE AUTHORS. THE REVISTA ELECTRÓNICA DE LEEME AND UNIVERSITAT DE VALĖNCIA ARE NOT LIABLE FOR ANY LEGAL ACTIONS THAT MAY ARISE INVOLVING THE ARTICLE'S CONTENT. REVISTA ELECTRÓNICA DE LEEME - ISTA ELECTRÓNICA EUROPEA DE MÚSICA EN LA EDUCACIÓN-HTTP://OJS.UV.ES/INDEX/PHP/LEEME/INDEX. ISSN: 1575-9563. EDITORES: UNIVERSIDAD DE VALENCIA Y JESÚS TEJADA GIMÉNEZ. VISIBILIDAD DE ESTA REVISTA: SCOPUS, EMERGING SOURCES CITATION INDEX (CLARIVATE), EBSCO, CINDOC (CSIC), CITEFACTOR, COPAC, DIALNET, DICE (CSIC), DOAJ, E-REVISTAS (CSIC), EBSCO PREMIER, ERIH+, GALE CENGAGE LEARNING, IN-RECS, IRESIE, LATINDEX, MIAR, OCLC WORLDCAT, RESH, REDIB, RILM CORE JOURNALS, SUDOC, ULRICHS, ESTA REVISTA ESTÁ PUBLICADA CON EL APOYO INSTITUCIONAL DE REDIRIS-CONSEJO SUPERIOR DE INVESTIGACIONES CIENTIFICAS Y ES DE ACCESO LIBRE. CREATIVE COMMONS LICENSE 4.O BY 
en un ítem de emociones, además de una tendencia a la significación en la puntuación total ( $p=.061$ ), y en un ítem de la estrategia basada en la técnica. En las dos primeras estrategias las diferencias fueron en la dirección de una mayor puntuación de las mujeres en la valoración de estas; en el caso del ítem de técnica fueron los hombres los que tuvieron puntuaciones superiores. Todos estos datos se resumen en la Tabla 1.

Tabla 1. Datos descriptivos y comparación entre mujeres y hombres

\begin{tabular}{|c|c|c|c|}
\hline & Mujeres & Hombres & \multirow{2}{*}{$t \circ z(p)^{*}$} \\
\hline & Media (DT) & Media (DT) & \\
\hline Modelado (puntuación total) & $11.97(3.42)$ & $12.62(3.45)$ & $1.3(.187)$ \\
\hline ¿Has estado alguna vez en una situación así? & $3.15(0.79)$ & $3.20(0.85)$ & $0.7(.510)$ \\
\hline ¿Si tuvieras que dar clases crees que lo harías de ese modo? & $2.28(0.83)$ & $2.44(0.90)$ & $1.2(.242)$ \\
\hline ¿Piensas que este método es correcto? & $2.38(0.88)$ & $2.55(0.93)$ & $1.3(.201)$ \\
\hline ¿Piensas que el estudiante aprenderá correctamente? & $2.29(0.81)$ & $2.39(0.88)$ & $0.6(.580)$ \\
\hline $\begin{array}{l}\text { ¿Crees que este es el mejor modo para mejorar la expresividad } \\
\text { musical? }\end{array}$ & $1.89(0.80)$ & $2.04(0.82)$ & $1.3(.198)$ \\
\hline Metáforas o imágenes (puntuación total) & $15.00(3.11)$ & $13.49(3.59)$ & $3.2(.002)$ \\
\hline ¿Has estado alguna vez en una situación así? & $3.16(0.97)$ & $2.59(1.01)$ & $4.0(<.001)$ \\
\hline ¿Si tuvieras que dar clases crees que lo harías de ese modo? & $2.989(0.82)$ & $2.69(0.93)$ & $1.4(.167)$ \\
\hline ¿Piensas que este método es correcto? & $3.12(0.66)$ & $2.83(0.84)$ & $2.5(.013)$ \\
\hline ¿Piensas que el estudiante aprenderá correctamente? & $2.95(0.71)$ & $2.71(0.80)$ & $2.0(.048)$ \\
\hline $\begin{array}{l}\text { ¿Crees que este es el mejor modo para mejorar la expresividad } \\
\text { musical? }\end{array}$ & $2.88(0.82)$ & $2.63(0.85)$ & $1.9(.062)$ \\
\hline Uso de las propias Emociones (puntuación total) & $13.73(3.42)$ & $12.75(3.86)$ & $1.9(.061)$ \\
\hline ¿Has estado alguna vez en una situación así? & $2.88(0.99)$ & $2.48(0.94)$ & $2.7(.006)$ \\
\hline ¿Si tuvieras que dar clases crees que lo harías de ese modo? & $2.74(0.83)$ & $2.58(0.94)$ & $1.2(.230)$ \\
\hline ¿Piensas que este método es correcto? & $2.82(0.75)$ & $2.61(0.90)$ & $1.7(.094)$ \\
\hline ¿Piensas que el estudiante aprenderá correctamente? & $2.67(0.78)$ & $2.56(0.87)$ & $0.9(.347)$ \\
\hline $\begin{array}{l}\text { ¿Crees que este es el mejor modo para mejorar la expresividad } \\
\text { musical? }\end{array}$ & $2.62(0.78)$ & $2.46(0.86)$ & $1.4(.177)$ \\
\hline Técnica (puntuación total) & $15.40(3.01)$ & $15.44(3.65)$ & $0.1(.934)$ \\
\hline ¿Has estado alguna vez en una situación así? & $3.35(0.76)$ & $3.18(0.90)$ & $1.2(.235)$ \\
\hline ¿Si tuvieras que dar clases crees que lo harías de ese modo? & $3.04(0.70)$ & $3.15(0.82)$ & $1.3(.181)$ \\
\hline ¿Piensas que este método es correcto? & $3.06(0.70)$ & $3.25(0.81)$ & $2.1(.034)$ \\
\hline ¿Piensas que el estudiante aprenderá correctamente? & $3.05(0.71)$ & $3.10(0.78)$ & $0.7(.493)$ \\
\hline $\begin{array}{l}\text { ¿Crees que este es el mejor modo para mejorar la expresividad } \\
\text { musical? }\end{array}$ & $2.87(0.77)$ & $2.74(0.97)$ & $0.8(.413)$ \\
\hline
\end{tabular}

-DT: desviación típica; resaltadas en negrita las comparaciones con diferencias estadísticamente significativas $(p<.05)$

* $t: t$ de Student con grados de libertad=N-2; $t$ es empleado en las comparaciones de medias para las puntuaciones totales; $z$ es el estadístico para las comparaciones no paramétricas mediante el test $U$ de Mann-Whitney sobre las distribuciones en cada grupo de los ítems específicos.

En los análisis de regresión lineal en los que la VD en cada análisis era la puntuación total en cada estrategia, la VI el género (con hombre como categoría de referencia) e incluyendo el país de la muestra como covariable (referencia: España) se encontró un efecto estadísticamente significativo para el género en el caso de metáforas o imágenes $(p=.002)$ y un

@CAROLINA BONASTRE Y ROBERTO NUEVO. THE CONTENT OF THIS ARTICLE IS THE SOLE RESPONSIBILITY OF THE AUTHORS. THE REVISTA ELECTRÓNICA DE LEEME AND UNIVERSITAT DE VALĖNCIA ARE NOT LIABLE FOR ANY LEGAL ACTIONS THAT MAY ARISE INVOLVING THE ARTICLE'S CONTENT, REVISTA ELECTRÓNICA DE LEEME -LISTA ELECTRÓNICA EUROPEA DE MÚSICA EN LA EDUCACIÓN-HTTP://OJS.UV.ES/INDEX/PHP/LEEME/INDEX. ISSN: 1575-9563. EDITORES: UNIVERSIDAD DE VALENCIA Y JESÚS TEJADA GIMÉNEZ. VISIBILIDAD DE ESTA REVISTA: SCOPUS, EMERGING SOURCES CITATION INDEX (CLARIVATE), EBSCO, CINDOC (CSIC), CITEFACTOR, COPAC, DIALNET, DICE (CSIC), DOAJ, E-REVISTAS (CSIC), EBSCO PREMIER, ERIH+, GALE CENGAGE LEARNING, IN-RECS, IRESIE, LATINDEX, MIAR, OCLC WORLDCAT, RESH, REDIB, RILM CORE JOURNALS, SUDOC, ULRICHS, ESTA REVISTA ESTÁ PUBLICADA CON EL APOYO INSTITUCIONAL DE REDIRIS-CONSEJO SUPERIOR DE INVESTIGACIONES CIENTIFICAS Y ES DE ACCESO LIBRE. CREATIVE COMMONS LICENSE 4.O BY 
efecto cercano al umbral de significación ( $p=.062$ ) en el caso de uso de las propias emociones: ser mujer se asoció con puntuaciones superiores en estas dos estrategias. El país de la muestra no tuvo pesos significativos en las ecuaciones de ambas, por lo que estos efectos pueden considerarse independientes del país de pertenencia. Estos resultados se resumen en la Tabla 2.

Tabla 2. Resultados de los análisis de regresión lineal (predicción de la puntuación en cada modelo en función del género

\begin{tabular}{lllll}
\hline Variables & $B(E T)$ & $t$ & $p$ & IC 95\% \\
\hline VD: Modelado & & & & \\
Género (ref.: Hombre=0) & $-0.663(0.49)$ & -1.35 & .177 & $-1.63,0.30$ \\
País (ref.: España=0) & $1.071(0.50)$ & 2.15 & .033 & $0.09,2.05$ \\
Constante & $12.196(0.40)$ & 30.70 & $<.001$ & $12.41,12.98$ \\
\hline VD: Metáforas o Imágenes & & & & \\
Género (ref.: Hombre=0) & $1.546(0.48)$ & 3.21 & .002 & $0.60,2.49$ \\
País (ref.: España=0) & $-0.465(0.49)$ & -0.95 & .344 & $-1.43,0.50$ \\
Constante & $13.644(0.39)$ & 34.81 & $<.001$ & $12.87,14.42$ \\
\hline VD: Uso de las propias emociones & & & & \\
Género (ref.: Hombre=0) & $0.986(0.52)$ & 1.88 & .062 & $-0.05,2.02$ \\
País (ref.: España=0) & $-0.483(0.53)$ & -0.90 & .367 & $-1.54,0.57$ \\
Constante & $12.496(0.43)$ & 28.86 & $<.001$ & $12.09,13.80$ \\
\hline VD: Técnica & & & & \\
Género (ref.: Hombre=0) & $-0.040(0.48)$ & -0.08 & .934 & $-0.99,0.91$ \\
País (ref.: España=0) & $-0.010(0.49)$ & -0.02 & .983 & $-0.98,0.96$ \\
Constante & $15.446(0.40)$ & 38.98 & $<.001$ & $14.66,16.23$ \\
\hline
\end{tabular}

Nota: $B$ : coeficiente de regresión; $E T$ : error típico; IC: Intervalo de Confianza para $B$.

Todos los análisis se repitieron considerando posibles diferencias de los resultados según el curso de las enseñanzas de cada estudiante y estos fueron equivalentes a los obtenidos para la muestra total.

\section{Discusión y conclusiones}

En un momento en el que la sociedad occidental, en general, concibe la igualdad de género como un aspecto importante y deseable es llamativo que existan preferencias tan marcadas en la elección de ciertos instrumentos musicales. Persiste una tendencia, desde hace tiempo, a que prevalezca la preferencia de los chicos hacia los instrumentos de viento-metal y percusión, destacando la tuba y una tendencia de las chicas hacia los instrumentos de vientomadera, destacando la flauta (Cramer, Million y Perreault, 2002; Sheldon y Price, 2005; Hallam, Rogers y Creech, 2008). También existe una predilección de los hombres hacia lo tecnológico y por tanto hacia instrumentos como la guitarra eléctrica y un interés más marcado de las mujeres hacia el canto (Green, 1997). Los resultados coinciden con los obtenidos en este trabajo, lo cual indica que estas diferencias se mantienen en el tiempo, al menos una década más tarde.

@CAROLINA BONASTRE Y ROBERTO NUEVO. THE CONTENT OF THIS ARTICLE IS THE SOLE RESPONSIBILITY OF THE AUTHORS. THE REVISTA ELECTRÓNICA DE LEEME AND UNIVERSITAT DE VALĖNCIA ARE NOT LIABLE FOR ANY LEGAL ACTIONS THAT MAY ARISE INVOLVING THE ARTICLE'S CONTENT, REVISTA ELECTRÓNICA DE LEEME -LISTA ELECTRÓNICA EUROPEA DE MÚSICA EN LA EDUCACIÓN-HTTP://OJS.UV.ES/INDEX/PHP/LEEME/INDEX. ISSN: 1575-9563. EDITORES: UNIVERSIDAD DE VALENCIA Y JESÚS TEJADA GIMÉNEZ. VISIBILIDAD DE ESTA REVISTA: SCOPUS, EMERGING SOURCES CITATION INDEX (CLARIVATE), EBSCO, CINDOC (CSIC), CITEFACTOR, COPAC, DIALNET, DICE (CSIC), DOAJ, E-REVISTAS (CSIC), EBSCO PREMIER, ERIH+, GALE CENGAGE LEARNING, IN-RECS, IRESIE, LATINDEX, MIAR, OCLC WORLDCAT, RESH, REDIB, RILM CORE JOURNALS, SUDOC, ULRICHS, ESTA REVISTA ESTÁ PUBLICADA CON EL APOYO INSTITUCIONAL DE REDIRIS-CONSEJO SUPERIOR DE INVESTIGACIONES CIENTIFICAS Y ES DE ACCESO LIBRE. CREATIVE COMMONS LICENSE 4.O BY 


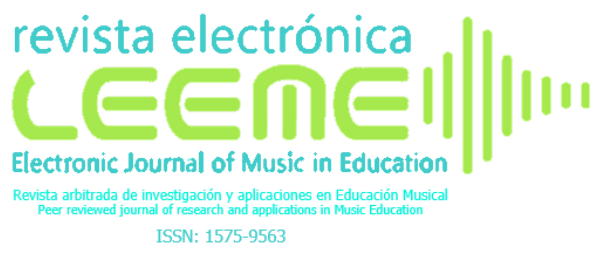

SECCIÓN MONOGRAFICO: CAROLINA BONASTRE Y ROBERTO NUEVO SECCION MONOGRAFICO: CAROLINA BONASTRE Y ROBERTO NUEVO
EL APRENDIZAJE EXPRESIVO Y LAS DIFERENCIAS DE GÉNERO EN LA EDUCACIÓN SUPERIOR DE
MÚSICA
(REVISTA ELECTRÓNICA DE LEEME) NÚMERO 44, PP. 87-101 $\frac{\text { HTTPS://OJS.UV.ES/INDEX.PHP/LEEME/INDEX }}{\text { DOI: } 10.7203 / \text { LEEME.44.15679 }}$

De acuerdo con Hallam et al. (2008) esto puede ser explicado por tres diferentes causas: a) factores sociales; b) características individuales; y c) la naturaleza del instrumento. Los factores sociales y culturales establecen expectativas estereotípicas de qué instrumentos son apropiados para cada género; y, por otro lado, los padres en la infancia y los compañeros en la adolescencia también influyen en las decisiones de los estudiantes. Del mismo modo hay que considerar la identidad musical individual, en lo que puede influir la edad de inicio: según la edad las preferencias de los estudiantes cambian en relación con los tipos de sonido y géneros musicales; la forma de interacción física con el instrumento también afecta a la elección, ya que los niños tienden a preferir instrumentos que son golpeados o requieren altos niveles de esfuerzo físico. Por último, existen disimilitudes claras según las características de algunos instrumentos posiblemente sumidas, en general, a las diferencias de registro, sonido, tamaño o aspectos particulares en la reproducción.

Los estudios muestran que estas experiencias se pueden cambiar poniendo en contacto a los niños con los diferentes instrumentos antes de su elección (Abeles y Porter, 1978) y dándoles a conocer experiencias de cambio de rol en dichos instrumentos, sobre todo para las mujeres (Bruce y Kemp, 1993). Quizás, dados los resultados, aún no son suficientes estas experiencias, además también podría ser aplicable a las preferencias profesionales.

Por otro lado, en nuestros días cada vez es mayor el número de compositoras y directoras profesionales, pero aún es un número notablemente inferior al de los hombres (Soler Campo, 2016). En los resultados se observa esta diferencia relevante en ambas preferencias profesionales. Aparte de los estereotipos sociales que existen en este campo (Soler Campo, 2016), se han encontrado desigualdades de género significativas en la creatividad musical (con puntuaciones altas en hombres) siendo su predictor más fuerte el ritmo (Lugo Mawang, Kigen y Mutweleli, 2019). También se ha encontrado que la asociación entre tecnología musical y composición en la clase de música de secundaria mejora la participación masculina (Green, 1997). Respecto a la dirección orquestal se ha establecido una relación estrecha con el papel del liderazgo (Soler Campo, 2016), que puede tener relación con los resultados. Lo que quizás sea más evidente para justificar la gran diferencia existente es que, aunque pudieran existir diferencias individuales, persisten los aspectos sociales que no facilitan esa intención educativa de igualdad de oportunidades.

Respecto a las estrategias de aprendizaje en la mejora de la expresividad hay que tener en cuenta que en gran medida dependerá de las propias concepciones que tenga el profesor sobre su importancia o sobre cómo mejorarla (Karlsson y Juslin, 2008). Los resultados sobre las opiniones de los estudiantes indican que las mujeres han aprendido recurriendo a metáforas e imágenes y enfocando su atención en sus emociones en mayor medida que los hombres. De hecho, parece que la influencia de cómo hemos aprendido es tan relevante que afecta a la elección de cómo enseñamos y quizás por ello en la práctica la mayoría están apegados a una

@CAROLINA BONASTRE Y ROBERTO NUEVO. THE CONTENT OF THIS ARTICLE IS THE SOLE RESPONSIBILITY OF THE AUTHORS. THE REVISTA ELECTRÓNICA DE LEEME AND UNIVERSITAT DE VAL ĖNCIA ARE NOT LIABLE FOR ANY LEGAL ACTIONS THAT MAY ARISE INVOLVING THE ARTICLE'S CONTENT. REVISTA ELECTRÓNICA DE LEEME -LISTA ELECTRÓNICA EUROPEA DE MÚSICA EN LA EDUCACIÓN-HTTP://OJS.UV.ES/INDEX/PHP/LEEME/INDEX. ISSN: 1575-9563. EDITORES: UNIVERSIDAD DE VALENCIA Y JESÚS TEJADA GIMÉNEZ. VISIBILIDAD DE ESTA REVISTA: SCOPUS, EMERGING SOURCES CITATION INDEX (CLARIVATE), EBSCO, CINDOC (CSIC), CITEFACTOR, COPAC, DIALNET, DICE (CSIC), DOAJ, E-REVISTAS (CSIC), EBSCO PREMIER, ERIH+, GALE CENGAGE LEARNING, IN-RECS, IRESIE, LATINDEX, MIAR, OCLC WORLDCAT, RESH, REDIB, RILM CORE JOURNALS, SUDOC, ULRICHS, ESTA REVISTA ESTÁ PUBLICADA CON EL APOYO INSTITUCIONAL DE REDIRIS-CONSEJO SUPERIOR DE INVESTIGACIONES CIENTIFICAS Y ES DE ACCESO LIBRE. CREATIVE COMMONS LICENSE 4.O BY 


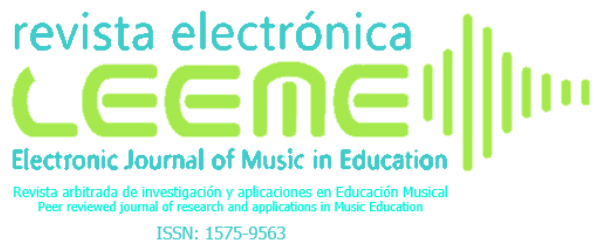

SECCIÓN MONOGRAFICO: CAROLINA BONASTRE Y ROBERTO NUEVO SECCIÓN MONOGRAFICO: CAROLINA BONASTRE Y ROBERTO NUEVO
EL APRENDIZAJE EXPRESIVO Y LAS DIFERENCIAS DE GÉNERO EN LA EDUCACIÓN SUPERIOR DE
MÚSICA (REVISTA ELECTRÓNICA DE LEEME) NÚMERO 44, PP. 87-101
HTTPS://OJS.UV.ES/INDEX.PHP/LEEME/INDEX

enseñanza tradicional (López-Iñiguez, Pozo y de Dios, 2013). En esta línea es natural que las participantes consideren que esas serán de forma general buenas estrategias de aprendizaje.

Por ello, se puede considerar razonable que haya una tendencia positiva, favorable a las mujeres, en el uso de la estrategia de elaboración de metáforas, en concordancia con otras investigaciones (Marugán et al., 2011). El presente estudio muestra que ellas consideran en mayor grado y de forma significativa que es un modo correcto para enseñar y que conlleva un aprendizaje efectivo. Del mismo modo, el hecho de enfatizar la importancia de esta estrategia puede tener relación con destacar, en dirección inversa y significativa, el menor acuerdo con la estrategia de técnica.

Las diferencias en función del género son independientes del país de la muestra. De todos modos, para el objetivo principal de este trabajo el país se entendía como una posible variable confusora que debía ser controlada. En este sentido, se partía de la hipótesis de que el país de procedencia no afectaría a las diferencias entre géneros. Los resultados encontrados apoyan esa idea. Las diferencias en las legislaciones educativas y, sobre todo, en la práctica real en el aula entre España y Reino Unido son, en todo caso, pequeñas (Müller, Norris, Hernández y Goodson, 2010), mientras que las similitudes culturales en general y sobre la perspectiva de género en particular son bastante claras. Valga como ejemplo la comparación del índice global de brecha de género del año 2018 del World Economic Forum que (con un rango de 0, máxima desigualdad de género, a 1, máxima igualdad) da una puntuación de 0.774 para Reino Unido y de 0.746 para España (World Economic Forum, 2018).

La limitación más relevante de este trabajo es el relativamente pequeño tamaño muestral, aunque las muestras pueden considerarse representativas en educación superior en música. Por otra parte, los resultados serían más concluyentes llevando a cabo estudios longitudinales al respecto en una más variada muestra internacional. También sería de interés indagar en las concepciones de los estudiantes desde una metodología mixta que permita aportar más información sobre el tema tratado en los diferentes niveles de especificación propuestos. Por otro lado, se remarca la importancia de la investigación sobre los procesos de enseñanzaaprendizaje desde esta perspectiva de género para determinar la consistencia de los resultados obtenidos.

Para disipar o disminuir las diferencias sociales y culturales, se proponen recomendaciones como la creación de planes de estudios inclusivos, con una práctica docente consciente de la influencia de los estereotipos de forma bidireccional que regule los posibles sesgos en el aula. También podría ser positivo enriquecer las salas de conciertos con la visión femenina del arte sonoro, mediante la programación de obras de grandes compositoras en centros universitarios y no universitarios para facilitar el acceso a los estudiantes. Sería conveniente que el repertorio femenino forme parte de los planes de estudios. De acuerdo con

@CAROLINA BONASTRE Y ROBERTO NUEVO. THE CONTENT OF THIS ARTICLE IS THE SOLE RESPONSIBILITY OF THE AUTHORS. THE REVISTA ELECTRÓNICA DE LEEME AND UNIVERSITAT DE VALĖNCIA ARE NOT LIABLE FOR ANY LEGAL ACTIONS THAT MAY ARISE INVOLVING THE ARTICLE'S CONTENT. REVISTA ELECTRÓNICA DE LEEME -LISTA ELECTRÓNICA EUROPEA DE MÚSICA EN LA EDUCACIÓN-HTTP://OJS.UV.ES/INDEX/PHP/LEEME/INDEX. ISSN: 1575-9563. EDITORES: UNIVERSIDAD DE VALENCIA Y JESÚS TEJADA GIMÉNEZ. VISIBILIDAD DE ESTA REVISTA: SCOPUS, EMERGING SOURCES CITATION INDEX (CLARIVATE), EBSCO, CINDOC (CSIC), CITEFACTOR, COPAC, DIALNET, DICE (CSIC), DOAJ, E-REVISTAS (CSIC), EBSCO PREMIER, ERIH+, GALE CENGAGE LEARNING, IN-RECS, IRESIE, LATINDEX, MIAR, OCLC WORLDCAT, RESH, REDIB, RILM CORE JOURNALS, SUDOC, ULRICHS, ESTA REVISTA ESTÁ PUBLICADA CON EL APOYO INSTITUCIONAL DE REDIRIS-CONSEJO SUPERIOR DE INVESTIGACIONES CIENTIFICAS Y ES DE ACCESO LIBRE. CREATIVE COMMONS LICENSE 4.O BY 


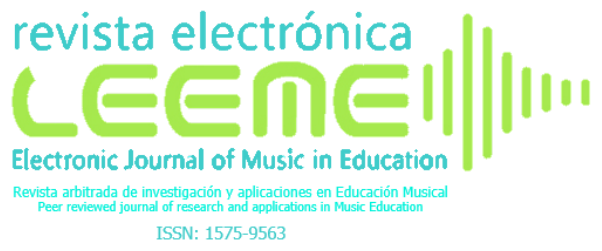

SECCIÓN MONOGRAFICO: CAROLINA BONASTRE Y ROBERTO NUEVO SECCION MONOGRAFICO: CAROLINA BONASTRE Y ROBERTO NUEVO
EL APRENDIZAJE EXPRESIVO Y LAS DIFERENCIAS DE GÉNERO EN LA EDUCACIÓN SUPERIOR DE
MÚSICA (REVISTA ELECTRÓNICA DE LEEME) NÚMERO 44, PP. 87-101
HTTPS://OJS.UV.ES/INDEX.PHP/LLEME/INDEX
DOI: $10.7203 /$ LEEME.44.15679

Bennett et al., (2018) que las instituciones promuevan la presencia de la mujer compositora y directora e intérprete (principalmente en los instrumentos donde persisten las diferencias de género) podría favorecer que en los entornos estudiantiles aumenten las oportunidades de las mujeres para considerar realmente todas sus posibilidades profesionales.

En síntesis, este trabajo muestra que la intervención de las mujeres en la composición y en la dirección es aún escasa debido seguramente a sus preferencias relacionadas con aspectos sociales y culturales. Respecto a los instrumentos que interpretan sigue habiendo una predilección por ciertos instrumentos y carencias en la visión femenina de especialidades instrumentales relacionadas principalmente con los instrumentos de viento-metal y percusión. Por otro lado, este estudio muestra la existencia de diferencias de género en la apreciación de estrategias de enseñanza-aprendizaje de la expresividad en la música: las mujeres se centran más en el recurso de la imaginación y la asociación con otros aspectos vinculantes para el estudiante como el uso de las emociones como conexión personal al propio aprendizaje. Sería importante desarrollar programas que, teniendo en cuenta estas diferencias, trabajen en la consecución de un cambio efectivo hacia la igualdad de oportunidades y en eludir e ir erradicando los estereotipos de género aún vigentes en la educación musical, incluso en niveles superiores de educación.

\section{Referencias}

Abeles, H.F y Porter, S.Y. (1978). The gender-stereotyping of musical instruments. Journal of Research in Music Education, 26(2), 65-75. doi: 10.2307/3344880

Agak, H. (2002). Gender difference and academic achievement in music among Form Four students in Kenya 1991-1994. Bulletin of the Council for Research in Music Education, 153/154(Spring \& Summer), 94-101. Recuperado de https://www.jstor.org/stable/40319147

Bonastre, C. y Timmers, R. (2019). Comparison of beliefs about teaching and learning of emotional expression in music performance between Spanish and English HE students of music. Psychology of Music. doi: 10.1177/0305735619842366

Bennett, D., Macarthur, S., Hope, C., Goh, T. y Hennekam, S. (2018). Creating a career as a woman composer: Implications for music in higher education. British Journal of Music Education, 35(3), 237-253. doi: 10.1017/S0265051718000104.

Brenner, B. y Strand, K. (2013). A case study of teaching musical expression to young performers. Journal of Research in Music Education, 61(1), 80-96. doi: $10.1177 / 0022429412474826$ 
Bruce, R. y Kemp, A (1993). Sex-stereotyping in children's preferences for musical instruments, British Journal of Music Education, 10(3), 213-17. doi: $10.1017 / \mathrm{S} 0265051700001777$

Cohen, J. (1994). The earth is round ( $p<.05)$. American Psychologist, 49(12), 997-1003.

Cramer, K.M., Million, E. y Perreault, L.A. (2002). Perception of musicians: Gender stereotypes and social role theory. Psychology of Music, 30(2), 164-174. doi: $10.1177 / 0305735602302003$

De las Heras Fernández, R. y García Gil, D. (2018). Estudio de los procesos de enseñanzaaprendizaje del flamenco a través de entrevistas. La visión de las mujeres. Revista Electrónica de LEEME, 43, 50-73. doi: 10.7203/LEEME.43.13587

Department for Education and Skills (2007). Gender and education: the evidence on pupils in England. Recuperado de https://www.education.gov.uk/publications/

Diaz Mohedo, M.T. (2005). La perspectiva de género en la formación del profesorado de música. Reice. Revista Electrónica Iberoamericana sobre Calidad, Eficacia y Cambio en Educación, 3(1), 570-577. Recuperado de http://www.ice.deusto.es/rinace/reice/vol3n1_e/Diaz.pdf

Eccles, J., Wigfield, A., Harold, R.D. y Blumenfeld, P. (1993). Age and gender differences in children's self- and task perceptions during elementary school. Child Development, 64(3), 830-47. doi: 10.2307/1131221

Gordon, E. (1986). Primary measures in music audiation. Chicago: GIA Publications.

Green, L. (1997). Music, gender and education. New York: Cambridge University Press.

Hallam, S., Rogers, L. y Creech, A. (2008). Gender differences in musical instrument choice. International Journal of Music Education, 26(1), 7-19. doi: $10.1177 / 0255761407085646$.

Hallam, S., Varvarigou y M., Creech, A., Papageorgi, I., Gomes, T., Lanipekun, J. y Tiija Rinta, T. (2017). Are there gender differences in instrumental music practice? Psychology of Music, 45(1), 116-130. doi: 10.1177/0305735616650994.

Juslin, P.N., Karlsson, J., Lindström, E.F., Friberg, A. y Schoonderwaldt, E. (2006). Play it again with feeling: Computer feedback in musical communication of emotions. Journal of Experimental Psychology: Applied, 12(2), 79-95. doi: 10.1037/1076-898X.12.2.79.

@CAROLINA BONASTRE Y ROBERTO NUEVO. THE CONTENT OF THIS ARTICLE IS THE SOLE RESPONSIBILITY OF THE AUTHORS. THE REVISTA ELECTRÓNICA DE LEEME AND UNIVERSITAT DE VALĖNCIA ARE NOT LIABLE FOR ANY CEGAL ACTIONS THAT MAY ARISE INVOLVING THE ARTICLE'S CONTENT REVISTA ELECTRÓNICA DE LEEME -LISTA ELECTRÓNICA EUROPEA DE MÚSICA EN LA EDUCACIÓN-HTTP://OJS.UV.ES/INDEX/PHP/LEEME/INDEX. ISSN: 1575-9563. EDITORES: UNIVERSIDAD DE VALENCIA Y JESÚS TEJADA GIMÉNEZ. VISIBILIDAD DE ESTA REVISTA: SCOPUS, EMERGING SOURCES CITATION INDEX (CLARIVATE), EBSCO, CINDOC (CSIC), CITEFACTOR, COPAC, DIALNET, DICE (CSIC), DOAJ, E-REVISTAS (CSIC), EBSCO PREMIER, ERIH+, GALE CENGAGE LEARNING, IN-RECS, IRESIE, LATINDEX, MIAR, OCLC WORLDCAT, RESH, REDIB, RILM CORE JOURNALS, SUDOC, ULRICHS, ESTA REVISTA ESTÁ PUBLICADA CON EL APOYO INSTITUCIONAL DE REDIRIS-CONSEJO SUPERIOR DE INVESTIGACIONES CIENTIFICAS Y ES DE ACCESO LIBRE. CREATIVE COMMONS LICENSE 4.O BY 
Juslin, P.N. y Laukka, P. (2004). Expression, perception, and induction of musical emotions: A review and a questionnaire study of everyday listening. Journal of New Music Research, 33(3), 217-238._doi: 10.1080/0929821042000317813

Karlsson, J. y Juslin, P.N. (2008). Musical expression: An observational study of instrumental teaching. Psychology of Music, 36(3), 309-334. doi:10.1177/0305735607086040.

Ley Orgánica 8/2013 para la mejora de la calidad educativa (2013). BOE 10 de mayo de 2013, 106, 97858-97921.

Lindström, E., Juslin, P.N., Bressin, R. y Williamon, A. (2003). Expressivity comes from within your soul: A questionnaire study of music students' perspectives on expressivity. Research Studies in Music Education, 20(1), 23-47. doi: $10.1177 / 1321103 X 030200010201$

López-Iñiguez, G., Pozo, J. y de Dios, M.J. (2013). The older, the wiser? Profiles of string instrument teachers with different experience according to their conceptions of teaching, learning, and evaluation. Psychology of Music, 42(2), 157-176. doi: $10.1177 / 0305735612463772$

Lugo Mawang, L., Kigen, E.M. y Mutweleli, S.M. (2019). The relationship between musical self-concept and musical creativity among secondary school music students. International Journal of Music Education, 37(1), 78-90. doi: $10.1177 / 0255761418798402$

Macarthur, S. (2010). Towards a Twenty-First-Century Feminist Politics of Music. New York: Ashgate.

Marugán de Miguelsanz, M., Catalina Sancho, J., Martín Antón, L.J., del Caño Sánchez, M., Hernández Ortega, C. y Carbonero Martín, M.A. (2011). Estrategias de elaboración en alumnos universitarios. International Journal of Developmental and Educational Psychology. INFAD Revista de Psicología, 1(3), 621-630. Recuperado de http://www.redalyc.org/articulo.oa?id=349832330065

Meissner, H. y Timmers, R. (2019). Teaching young musicians expressive performance: An experimental study. Music Education Research, 21(1), 20-39. doi: 10.1080/14613808.2018.1465031.

Müller, J., Norris, C., Hernández, F. y Goodson, I. (2010). Restructuring teachers' work-lives and knowledge in England and Spain. Compare, 40(3), 265-277. doi: $10.1080 / 03057920902830061$

@CAROLINA BONASTRE Y ROBERTO NUEVO. THE CONTENT OF THIS ARTICLE IS THE SOLE RESPONSIBILITY OF THE AUTHORS. THE REVISTA ELECTRÓNICA DE LEEME AND UNIVERSITAT DE VALĖNCIA ARE NOT LIABLE FOR ANY LEGAL ACTIONS THAT MAY ARISE INVOLVING THE ARTICLE'S CONTENT, REVISTA ELECTRÓNICA DE LEEME —LISTA ELECTRÓNICA EUROPEA DE MÚSICA EN LA EDUCACIÓN-HTTP://OJS.UV.ES/INDEX/PHP/LEEME/INDEX. ISSN: 1575-9563. EDITORES: UNIVERSIDAD DE VALENCIA Y JESÚS TEJADA GIMÉNEZ. VISIBILIDAD DE ESTA REVISTA: SCOPUS, EMERGING SOURCES CITATION INDEX (CLARIVATE), EBSCO, CINDOC (CSIC), CITEFACTOR, COPAC, DIALNET, DICE (CSIC), DOAJ, E-REVISTAS (CSIC), EBSCO PREMIER, ERIH+, GALE CENGAGE LEARNING, IN-RECS, IRESIE, LATINDEX, MIAR, OCLC WORLDCAT, RESH, REDIB, RILM CORE JOURNALS, SUDOC, ULRICHS, ESTA REVISTA ESTÁ PUBLICADA CON EL APOYO INSTITUCIONAL DE REDIRIS-CONSEJO SUPERIOR DE INVESTIGACIONES CIENTIFICAS Y ES DE ACCESO LIBRE. CREATIVE COMMONS LICENSE 4.O BY 
Müllensiefen, D., Gingras, B., Musil, J. y Stewart, L. (2014). The musicality of non-musicians: an index for assessing musical sophistication in the general population. Plos One, 9, e101091. doi: 10.1371/journal.pone.0089642

Parsons, L. y Ravenscroft, B. (2016). Analytical Essays on Music by Women Composers: Concert Music, 1960-2000. Oxford: Oxford University Press.

Raglio, A., Bellandi, D., Gianotti, M., Zanacchi, E., Gnesi, M., Monti, M.C. e Imbriani, M. (2019). Daily music listening to reduce work-related stress: a randomised controlled pilot trial. Journal of Public Health. Prepublicación online. doi:10.1093/pubmed/fdz030

Sheldon, D.A. y Price, H.E. (2005). Gender and Instrumentation distribution in an international cross-section of wind and percussion ensembles. Bulletin of the Council for Research in Music Education, 163, 43-51. Recuperado de https://www.jstor.org/stable/40311594

Sloboda, J. A., Gayford, C. y Minassian, C. (2003, septiembre). Assisting advanced musicians to enhance their expressivity: An intervention study. Communication presented at the Fifth Triennial Conference of the European Society for the Cognitive Sciences of Music, Hannover.

Soler Campo, S. (2016). Mujeres y música. Obstáculos vencidos y caminos por recorrer. Dossiers Feministes, 21, 157-174. doi:10.6035/Dossiers.2016.21.10.

Williamon, A. (2014). Implications for education. En D. Fabian, R. Timmers y E. Schubert, Expressiveness in music performance. Empirical approaches across styles and cultures (pp.348-352). Oxford: Oxford University Press.

Woody, R.H. (2006). The effect of various instructional conditions on expressive music performance. Journal of Research and Music Education, 54(1), 21-36. doi:10.1177/002242940605400103

World Economic Forum (2018). The Global Gender Gap Report. 2018. Ginebra: World Economic Forum.

Young, V, Burwell, K. y Pickup, D. (2003). Areas of study and teaching strategies in instrumental teaching: A case study research project. Music Education Research, 5(2), 139-155. doi: 10.1080/1461380032000085522

Zervoudakes, J. y Tanur, J. (1994). Gender and musical instruments: Winds of change. Journal of Research in Music Education, 42(1), 58-67. doi: 10.2307/3345337

@CAROLINA BONASTRE Y ROBERTO NUEVO. THE CONTENT OF THIS ARTICLE IS THE SOLE RESPONSIBILITY OF THE AUTHORS. THE REVISTA ELECTRÓNICA DE LEEME AND UNIVERSITAT DE VALĖNCIA ARE NOT LIABLE FOR ANY LEGAL ACTIONS THAT MAY ARISE INVOLVING THE ARTICLE'S CONTENT. REVISTA ELECTRÓNICA DE LEEME -LISTA ELECTRÓNICA EUROPEA DE MÚSICA EN LA EDUCACIÓN-HTTP://OJS.UV.ES/INDEX/PHP/LEEME/INDEX. ISSN: 1575-9563. EDITORES: UNIVERSIDAD DE VALENCIA Y JESÚS TEJADA GIMÉNEZ. VISIBILIDAD DE ESTA REVISTA: SCOPUS, EMERGING SOURCES CITATION INDEX (CLARIVATE), EBSCO, CINDOC (CSIC), CITEFACTOR, COPAC, DIALNET, DICE (CSIC), DOAJ, E-REVISTAS (CSIC), EBSCO PREMIER, ERIH+, GALE CENGAGE LEARNING, IN-RECS, IRESIE, LATINDEX, MIAR, OCLC WORLDCAT, RESH, REDIB, RILM CORE JOURNALS, SUDOC, ULRICHS, ESTA REVISTA ESTÁ PUBLICADA CON EL APOYO INSTITUCIONAL DE REDIRIS-CONSEJO SUPERIOR DE INVESTIGACIONES CIENTIFICAS Y ES DE ACCESO LIBRE. CREATIVE COMMONS LICENSE 4.O BY 